\section{Kidney \\ Blood Pressure Research}

\title{
HDAC Inhibition Suppresses Cardiac Hypertrophy and Fibrosis in DOCA-Salt Hypertensive Rats via Regulation of HDAC6/HDAC8 Enzyme Activity
}

\author{
Hae Jin Kee Eun Hui Bae $^{\mathrm{b}}$ Sangha Park ${ }^{\mathrm{a}}$ Ko Eun Lee ${ }^{\mathrm{b}}$ Sang Heon Suh ${ }^{\mathrm{b}}$ \\ Soo Wan Kim ${ }^{b}$ Myung Ho Jeong ${ }^{\mathrm{a}}$ \\ a Heart Research Center of Chonnam National University Hospital, and ${ }^{b}$ Department of Internal \\ Medicine, Chonnam National University Medical School, Gwangju 501-757, Republic of Korea
}

\section{Key Words}

Histone deacetylase inhibitor • GATA6 • HDAC enzyme activity • Cardiac hypertrophy • DOCA-salt hypertensive rats

\begin{abstract}
Background: Inhibition of histone deacetylase (HDAC) was reported to suppress cardiac hypertrophy and fibrosis in various hypertrophic animal models. However, the HDAC expression profile and HDAC enzyme activity have not yet been investigated in DOCA-salt hypertensive rats. Methods: Unilaterally nephrectomized rats were implanted with DOCA strips. DOCAsalt rats then received a control diet with vehicle or valproate. We measured the expression of cardiac hypertrophic markers, class I HDACs, class II HDACs, fibrosis, and HDAC enzyme activity. Results: Here we report that sodium valproate inhibits the cardiac hypertrophy accompanied by fibrosis in the heart of chronic hypertensive rats. We show that expression of GATA6 and HDAC6 is upregulated in DOCA-salt hypertension. In addition, HDAC6 and HDAC8 enzyme activity is attenuated by sodium valproate. Conclusion: These results suggest that a novel HDAC6- and HDAC8-selective inhibitor is needed to treat or prevent pathological cardiac hypertrophy.
\end{abstract}

Copyright $@ 2013$ S. Karger AG, Basel

\section{Introduction}

Histone deacetylases (HDACs) catalyze the removal of the acetyl group from histone residues and play a key role in modulating gene expression. HDACs are classified into four distinct classes on the basis of similarity to yeast transcriptional repressors [1]. Class I HDACs (HDACs 1, 2, 3, and 8) promote pathological cardiac hypertrophy, whereas class

Hae Jin Kee, PhD, and Myung Ho Jeong, $\mathrm{MD}, \mathrm{PhD}$, Director of Heart Research

Center Nominated by Korea Ministry of Health and Welfare
Heart Research Center of Chonnam National University Hospital, Chonnam National University Hospital, 671 Jebong-ro, Dong-gu, Gwangju 501-757 (Republic of Korea) Tel. +82-62-220-5823 / and -6243, Fax +82-62-228-4227 / and -7174

E-Mails sshjkee@hanmail.net and myungho@chollian.net 


\section{Kidney \\ Blood Pressure Research}

Kidney Blood Press Res 2013;37:229-239

\begin{tabular}{l|l}
\hline DOI: 10.1159/000350148 & (c) 2013 S. Karger AG, Basel
\end{tabular}

Published onlıne: July 8, 2013

www.karger.com/kbr

Kee/Bae/Park/Lee/Suh/Kim/Jeong: Valproic Acid Inhibits Cardiac Hypertrophy and Fibrosis

IIa HDACs (HDACs 4, 5, 7, and 9) suppresses cardiac hypertrophy. The role of the class IIb HDACs (HDAC6 and HDAC10) remains unknown. Class III HDACs (sirtuins 1-7) function as cardioprotective factors. The role of the class IV HDAC11 is unknown.

Dysregulation of HDACs is associated with various pathophysiological processes, including cancer, neurological disorders, inflammation, cardiac hypertrophy, and heart failure. Recent studies have revealed an inhibitory effect of HDAC inhibitors on cardiac hypertrophy and heart failure as well as on restenosis after balloon angioplasty or stent implantation [2, 3]. Our previous report showed that, among the class I HDACs, activation of HDAC2 enzyme by heat shock protein 70 (Hsp70) can trigger cardiac hypertrophy in response to diverse hypertrophic stimuli [4]. Recently, another group observed that HDAC6 catalytic activity is induced in rats in response to chronic hypertension induced by deoxycorticosterone acetate (DOCA)-salt [5].

Cardiac hypertrophy is the enlargement of the heart in response to physiological or pathological stimuli. Prolonged cardiac hypertrophy is associated with arrhythmia, sudden death, decompensation, and dilated cardiomyopathy and results in heart failure [6]. Cardiac hypertrophy is accompanied by the reactivation of fetal gene expression, an increase in protein synthesis, and an increase in stress fiber formation. Interstitial fibrosis is a hallmark of adverse cardiac remodeling in response to stress such as hypertension, valve disease, or myocardial infarction. In particular, chronic pathological stress often leads to excessive extracellular matrix (ECM) protein deposition in the left ventricle of the heart. ECM accumulation is mediated by transforming growth factor- $\beta$ (TGF- $\beta$ ) through the Smad signaling pathway.

Left ventricular hypertrophy is found in most animal models of hypertension. For example, DOCA-salt hypertensive rats show cardiac hypertrophy. We and other research teams have reported that HDAC inhibitors such as trichostatin A, sodium valproate, and butyrate can prevent cardiac hypertrophy in animal models in response to various hypertrophic stimuli $[2,7,8]$. However, study of the HDAC inhibition of cardiac hypertrophy and fibrosis has not been conducted in the DOCA-salt hypertensive rat model. In this study, therefore, we investigated whether sodium valproate can prevent cardiac hypertrophy and fibrosis in DOCA-salt hypertensive rats. We also examined the mechanism by which valproate inhibits cardiac hypertrophy and fibrosis in the left ventricle of DOCA-salt hypertensive rats.

\section{Materials and Methods}

\section{Materials and antibodies}

Deoxycorticosterone acetate was purchased from Sigma (St. Louis, MO, USA). Sodium valproate was purchased from Sigma (St. Louis, MO, USA). Western blot was performed with antibodies against Gapdh (sc-32233, Santa Cruz), Nkx-2.5 (sc-376565), SRF (sc-335), HDAC1 (sc-7872), HDAC2 (sc-7899), HDAC3 (sc-11417), HDAC4 (sc-11418), HDAC6 (sc-5258), and HDAC8 (sc-11405). HDAC5 (\#2082p) and HDAC7 (\#2882p) were obtained from Cell Signaling (Cell Signaling Technology, Inc., Danvers, MA, USA). HDAC9 (ab18970), GATA4 (ab84593), GATA6 (ab39123), Type I collagen (ab34710), and MEF2C (ab64644) were purchased from Abcam (Abcam, Cambridge, UK). ANF (E54066M) was obtained from Meridian (Meridian Life Science, Inc, Saco, ME, USA). Wheat germ agglutinin Alexa Fluor 488 conjugate was purchased from Invitrogen (Molecular Probes).

DOCA-salt hypertensive rat model

The animal study was approved by the Ethics Committee of Chonnam National University Medical School. Male Sprague-Dawley rats weighing 180 to $200 \mathrm{~g}$ were used. The DOCA-salt hypertension model was prepared as previously described [9]. 


\section{Kidney \\ Blood Pressure Research}

Kidney Blood Press Res 2013;37:229-239

\begin{tabular}{l|l}
\hline DOI: $10.1159 / 000350148$ & (c) 2013 S. Karger AG, Basel
\end{tabular}

Published onine: July 8, 2013

www.karger.com/kbr

DOCA-salt hypertension was induced by subcutaneous implantation of silicone rubber containing DOCA (200 mg/kg) at 1 week after unilateral nephrectomy. Physiological saline was supplied as the drinking water to all animals. Two weeks after DOCA implantation, DOCA-salt hypertensive rats were randomly divided to receive a control diet with or without sodium valproate $(0.71 \% \mathrm{wt} / \mathrm{vol})$ for another 2 weeks.

Reverse transcription-polymerase chain reaction (RT-PCR)

Total RNA was isolated with TriZol reagent (Invitrogen Life Technologies), and $1 \mu \mathrm{g}$ of RNA was used for the reverse transcription reaction with the Superscript first-strand synthesis system for RT-PCR kit (Invitrogen Life Technologies). Quantification of mRNA amounts was done with the SYBR Green PCR kit (Applied Biosystems, Inc). PCR was performed by using the following oligonucleotide primers: for Nppa, sense, 5'-GCTCGAGCAGATCGCAAAAG-3', and antisense, 5'-GAGTGGGAGAGGTAAGGCCT-3' ; for Nppb, sense, 5'-TAATCTGTCGCCGCTGGGAGG-3', and antisense, 5'-CAGCTGGGGAAAGAAGAGCCG-3'; for Myh7, sense, 5'-ACAGAGGAAGACAGGAAGAACCTACTG-3', and antisense, 5'-GCACAAGAT-CTACTCCTCATTCAGG-3'; for collagen type I, sense, 5'-CTCCTGACGCATGGCCAAGA-3', and antisense, 5'-TGGGTCCCTCGACTCCTATG-3'; for fibronectin, sense, 5'-AGCAAATCG-TGCAGCCTCCG-3', and antisense, 5'-CCCCCTTCATGGCAGCGATT-3'; for CTGF, sense, 5'- TCCCGTTAGCCTCGCCTTGG-3', and antisense, 5'- CGGTACACGGACCCACCGAA-3'; for Gapdh, sense, 5'-TGCACCACCAACTGCTTAG-3', and antisense, 5'-GATGCAGGGATGATGTTC-3'.

\section{Western blot analysis}

Western blots were performed as described previously [10]. Cells lysates were prepared with RIPA buffer $(150 \mathrm{mM} \mathrm{NaCl}, 1 \%$ Triton X-100, 1\% sodium deoxycholate, $50 \mathrm{mM}$ tris- $\mathrm{HCl}, \mathrm{pH}$ 7.5, $2 \mathrm{mM}$ EDTA, $5 \mathrm{mM} \mathrm{NaF}$ ) containing protease inhibitors. Proteins were separated by $8 \sim 12 \%$ SDS-PAGE and were then transferred to polyvinylidene difluoride (PVDF) membranes. The membranes were probed with the indicated antibodies and developed by using Immobilon Western Detection Reagents (Millipore, Billerica, MA, USA).

\section{Assessment of cardiomyocyte size and immunohistochemistry}

Paraffin-embedded sections of left ventricle were cut into $6-\mu \mathrm{m}$ sections and stained with wheat germ agglutinin Alexa Fluor 488 (1:200), which stains the cell membrane. Images were captured by using NIS Elements Imaging Software (Nikon, Japan) to determine the cross-sectional area of cardiac myocytes.

For immunohistochemistry, perfusion was performed as described previously [11]. Heart sections of DOCA-salt rats (6- $\mu \mathrm{m}$ thick) were immunostained with anti-type I collagen (1:100). To reduce autofluorescence, heart sections were treated with $0.1 \%$ Sudan Black B stain.

HDAC activity assay

HDAC activity was determined by using the Fluor-de-Lys HDAC activity assay kit (Biomol) as described previously [4]. After heart tissues were lysed with RIPA buffer, HDAC activity was measured by immunoprecipitation assay by use of the respective antibodies (HDAC1, HDAC2, HDAC3, HDAC6, and HDAC8).

\section{Statistical analysis}

Statistical analysis was performed with either Student's $t$-test or one-way ANOVA with a Bonferroni post hoc test with software provided by GraphPad Prism version 5.0. Data are presented as means \pm S.E.M. A P value of $<0.05$ was considered statistically significant.

\section{Results}

Valproate inhibits cardiac hypertrophy in DOCA-salt hypertensive rats

To determine whether the DOCA-salt hypertensive rats showed heart enlargement, we determined the heart weight/body weight (HW/BW) ratio. The HW/BW ratio was higher in the DOCA-salt hypertensive rats than in sham control rats, whereas treatment with valproate significantly suppressed the HW/BW ratio in the DOCA-salt hypertensive rats (Fig. 1a). Wheat 


\section{Kidney Blood Pressure Research}

Fig. 1. Valproate reverses cardiac hypertrophy in DOCA-salt hypertensive rats. a Heart weight to body weight (HW/BW) ratios in sham rats, DOCA-salt hypertensive rats (DOCA), and DOCA-salt hypertensive rats treated with valproate (DOCA+VAL) 4 weeks after DOCA-salt treatment. Values are expressed as mean \pm SE. $b$ Myocyte size as measured by cross-sectional areas at 4 weeks. c Wheat germ agglutinin staining showing cell size in the left ventricle at 4 weeks. d Representative RT-PCR analysis of heart mRNA from sham, DOCA, and DOCA+VAL rats. The transcript levels of Nppa (ANF), Nppb (BNP), and Myh7 were evaluated. e-g Quantitative analysis of changes in Nppa, Nppb, and Myh7 mRNA amounts by real-time PCR. GAP-

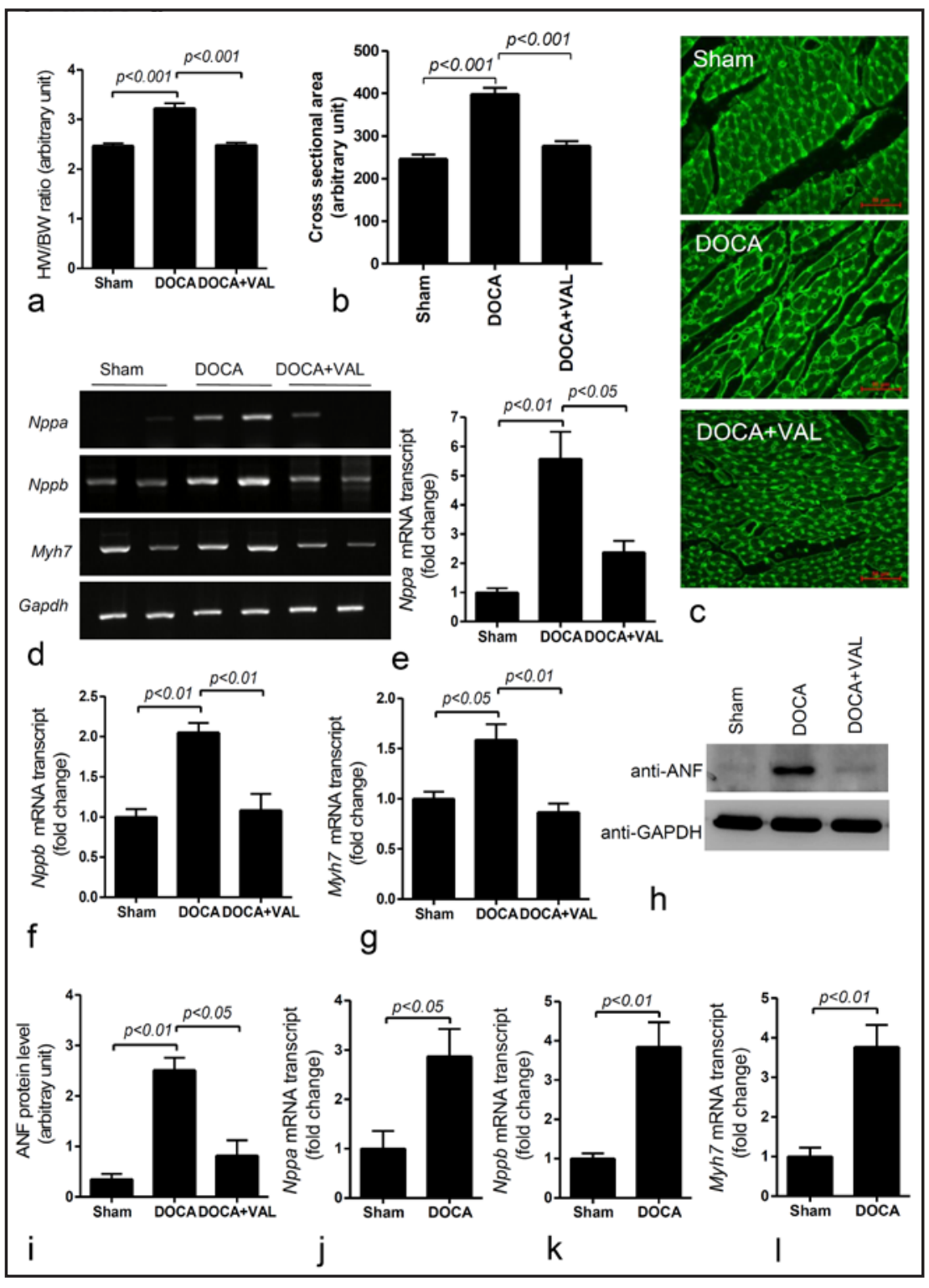

DH was used as an internal control. Four to five hearts from each experiment were assessed. h Representative immunoblot analysis of ANF and GAPDH in the rat hearts treated with VAL. Antibody against GAPDH was used as a loading control. i Quantitative analysis of ANF from Fig. 1h. j-l Quantitative analysis of changes in Nppa, Nppb and Myh7 mRNA amounts by real-time PCR at 2 weeks after DOCA-salt treatment.

germ agglutinin staining to evaluate cardiac myocyte size showed a significant increase in cross-sectional area in DOCA-salt hypertensive rats compared with sham rats (Fig.1b and 1c). Expectedly, cardiac myocytes of valproate-treated DOCA-salt hypertensive rats revealed a decrease in cell size in the left ventricle. To further examine whether valproate affected markers of hypertrophic expression, we performed qRT-PCR in heart tissue. The transcript levels of $N p p a$ (ANF), Nppb (BNP), and Myh7 ( $\beta$-MHC) in rat hearts were significantly enhanced in the DOCA-salt hypertensive rats compared with the levels in sham-operated rats. Furthermore, these increases were inhibited by administration of valproate (Fig. 1d-g).

Next, we checked whether valproate affected the protein levels of ANF in the DOCA-salt hypertensive rats. As shown in Fig. $1 \mathrm{~h}$ and 1i, protein levels of ANF were increased in DOCAsalt hypertensive rats, and this increase was significantly attenuated by treatment with 


\section{Kidney \\ Blood Pressure Research}

Fig. 2. GATA6 protein expression is induced in the heart of DOCA-salt hypertensive rats. a Cardiac transcription factors including GATA4, GATA6, MEF2C, and SRF were determined by Western blot analysis by using hearts from sham rats, DOCA-salt hypertensive rats (DOCA), and DOCA-salt hypertensive rats treated with valproate (DOCA+VAL). GAPDH was used as a loading control. GATA4 (b), GATA6 (c), MEF2C (d), and SRF (e) protein were quantified. Values are expressed as mean \pm SE.
Kidney Blood Press Res 2013;37:229-239

\begin{tabular}{l|l}
\hline DOI: $10.1159 / 000350148$ & (c) 2013 S. Karger AG, Basel
\end{tabular}

Published onlıne: July 8, 2013

www.karger.com/kbr

233

Kee/Bae/Park/Lee/Suh/Kim/Jeong: Valproic Acid Inhibits Cardiac Hypertrophy and Fibrosis

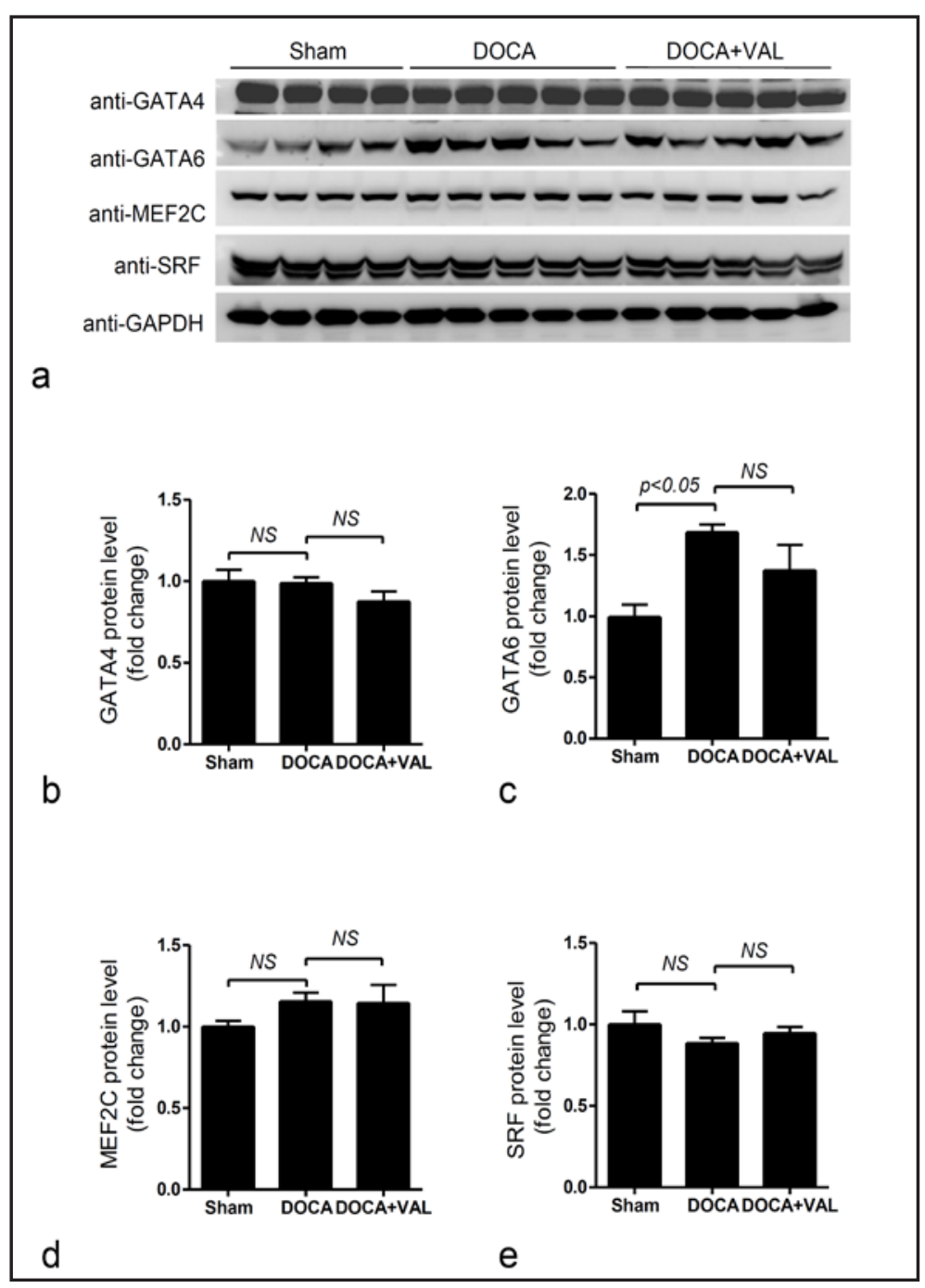

valproate. We also investigated whether the inhibitory effect of valproate was to reverse or prevent cardiac hypertrophy by studying the expression of cardiac hypertrophic markers at 2 weeks after DOCA treatment. Expression of $N p p a, N p p b$, and $M y h 7$ was significantly increased at 2 weeks after DOCA-salt treatment compared with that in sham rats (Fig. 1j-1). These results suggested that valproate reversed the cardiac hypertrophy induced by DOCAsalt.

Transcription factor GATA6 protein is increased in DOCA-salt hypertensive rats

Cardiac transcription factors are known to play a crucial role in mediating cardiac gene transcription in response to hypertrophic stimuli [12]. To identify whether cardiac-specific genes are affected in DOCA-salt hypertensive rats, we performed Western blot analysis. As shown in Fig. 2, the protein levels of GATA4, MEF2C, and SRF were not changed among the groups. In contrast, GATA6 protein was expressed more in hearts from the DOCA-salt hypertensive rats compared with hearts from the control rats. However, this increase was not blocked by valproate treatment.

Class I and class IIa HDAC protein levels are not changed except for HDAC6 in DOCA-salt hypertensive rats

HDAC inhibitors have been shown to affect HDAC enzyme activity in animal models of pathological hypertrophy. In this study, we first checked whether hypertensive stress or 


\section{Kidney Blood Pressure Research}

Fig. 3. Class I and II HDAC protein levels except for HDAC6 are not changed in DOCA-salt hypertensive rats. a Protein expression of class I HDACs, including HDAC1, HDAC2, HDAC3, and HDAC8, was determined by Western blot analysis using hearts from sham rats, DOCAsalt hypertensive rats (DOCA), and DOCA-salt hypertensive rats treated with valproate (DOCA+VAL). GAPDH was used as a loading control. b Quantitative results from panel A. c Class IIa (HDAC4, HDAC5, HDAC7, and HDAC9) and class IIb (HDAC6) protein expression was evaluated by Western blot analysis. $\mathrm{d}$ HDAC6 protein was induced in the heart of DOCA-salt hyper-

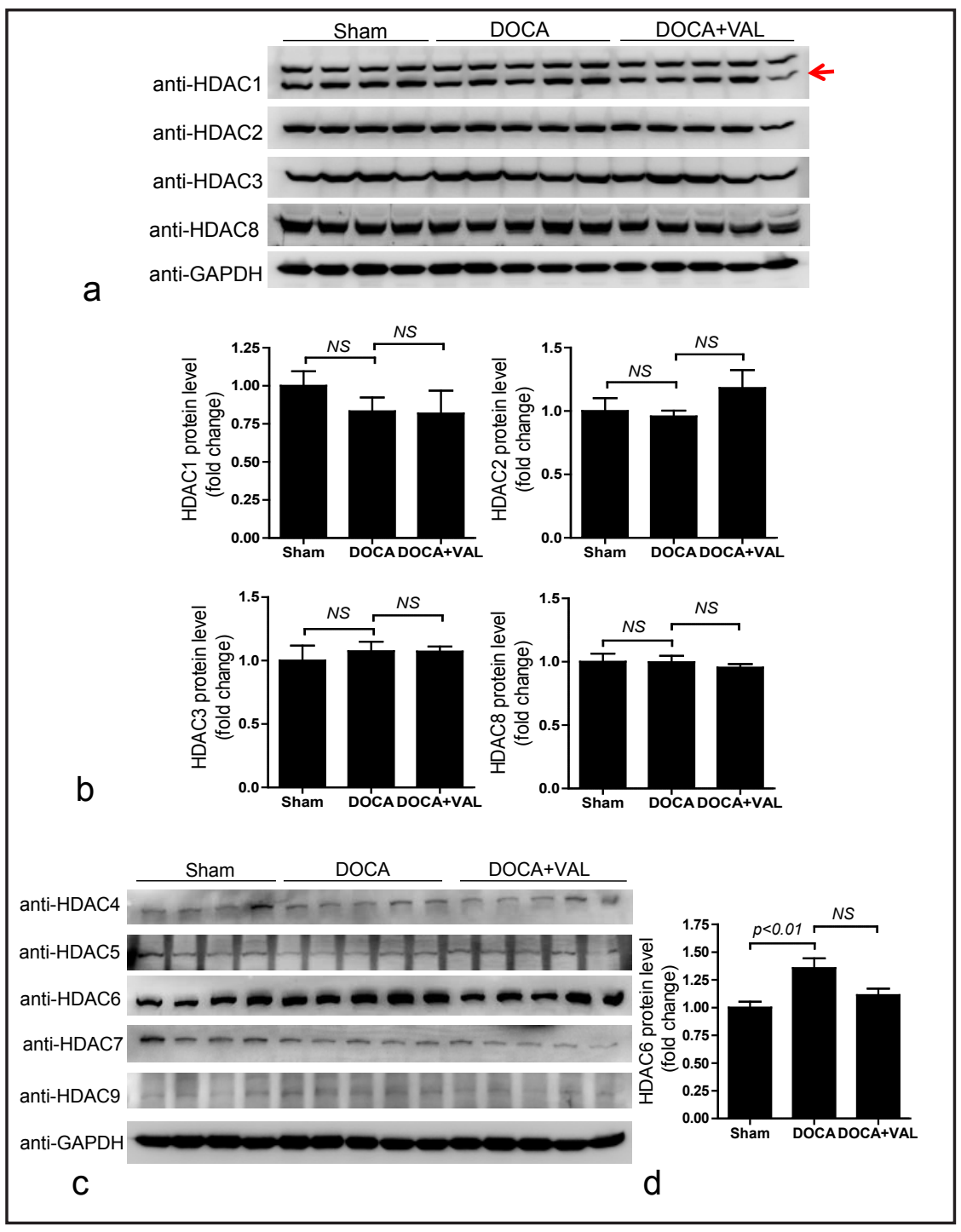
tensive rats.

HDAC inhibitor could regulate HDAC expression. As shown in Fig. $3 a$ and $3 b$, the expression of class I HDACs including HDAC1, HDAC2, HDAC3, and HDAC8 was not changed at the protein level in the different groups. Next, we investigated the protein expression of the class II HDACs, including HDAC4, HDAC5, HDAC6, HDAC7, and HDAC9. Of those, HDAC6 protein expression was significantly increased in hearts of DOCA-salt hypertensive rats (Fig. $3 \mathrm{c}$ and $3 d)$.

HDAC6 and HDAC8 enzyme activity induced by DOCA-salt hypertension is inhibited by valproate

We previously reported that HDAC2 enzyme activity is induced at the early stages of cardiac hypertrophy [4]. In this study, we investigated whether total HDAC activity is affected in hypertrophied hearts induced by DOCA-salt hypertension. As shown in Fig. 4a, we found that global HDAC activity was largely increased in hearts of DOCA-salt hypertensive rats, and that this increase was suppressed in rats treated with valproate. Next, we examined HDAC enzyme activity with specific antibodies to determine which type of HDAC is activated in response to DOCA-salt hypertension. Unexpectedly, we found that the enzyme activity 


\section{Kidney Blood Pressure Research}

Fig. 4. HDAC6 and HDAC8 enzyme activity are induced by DOCA-salt hypertension. a Global HDAC activity in sham rats, DOCA-salt hypertensive rats (DOCA), and DOCA-salt hypertensive rats treated with valproate (DOCA+VAL) was measured by using a colorimetric HDAC assay kit as described in the Methods. b-f Class I HDACs (HDAC1, HDAC2, HDAC3, and HDAC8) and class IIb (HDAC6) activity in the heart was evaluated by immunoprecipitation assay by use of the respective antibodies. HDAC6 and HDAC8 enzyme activity induced by DOCA-salt hypertension was attenuated by valproate.

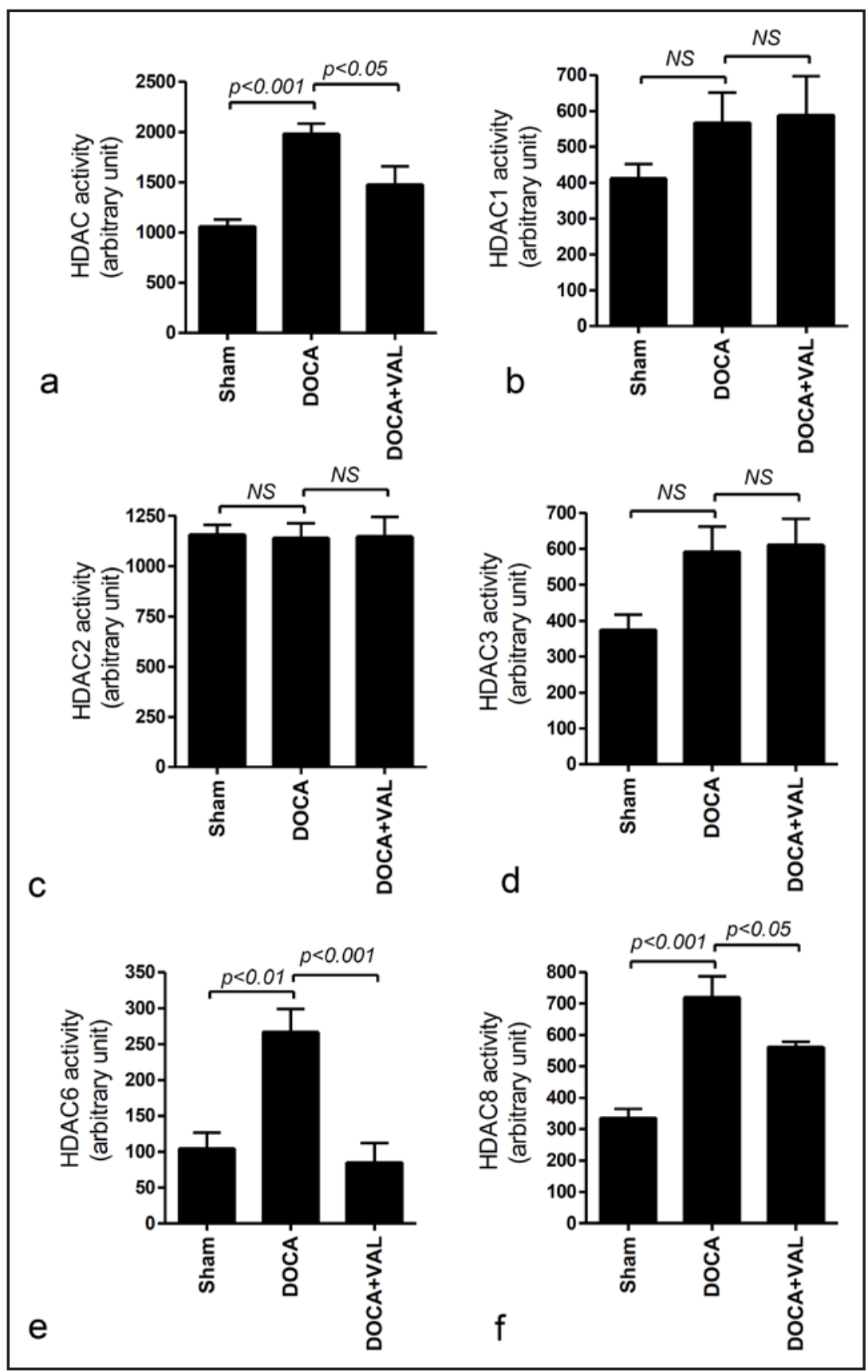

of HDAC8 (Fig. 4f) but not that of HDAC2 (Fig. 4c) was significantly induced in the heart of DOCA-salt hypertensive rats and was attenuated with valproate treatment. Although other class I HDACs such as HDAC1 (Fig. 4b) and HDAC3 (Fig. 4d) did not show a significant increase in DOCA-salt hypertensive rats, they did seem to be partly increased. Furthermore, we tested whether a class IIb such as HDAC6 (Fig. 4e) is affected in DOCA-salt hypertensive rats. We found that the enzyme activity of HDAC6 was significantly increased in DOCA-salt hypertensive rats and that the increase was completely blocked by sodium valproate.

Valproate inhibits fibrosis in the hearts of DOCA-salt hypertensive rats

Cardiac fibrosis is the result of numerous pathological processes in response to mechanical, neurohormonal stimuli and is related to hypertension in patients with left 


\section{Kidney Blood Pressure Research}

Fig. 5. Valproate suppresses cardiac fibrosis in DOCA-salt hypertensive rats. a Representative gel pictures are shown. Real time RT-PCR analysis for fibrosis markers including collagen type I (b), fibronectin (c), and CTGF (d) was performed. e Masson's trichrome staining in rat hearts at 4 weeks. Interstitial fibrosis is shown in blue. $\mathrm{f}$ Representative images of type I collagen deposition in the hearts.

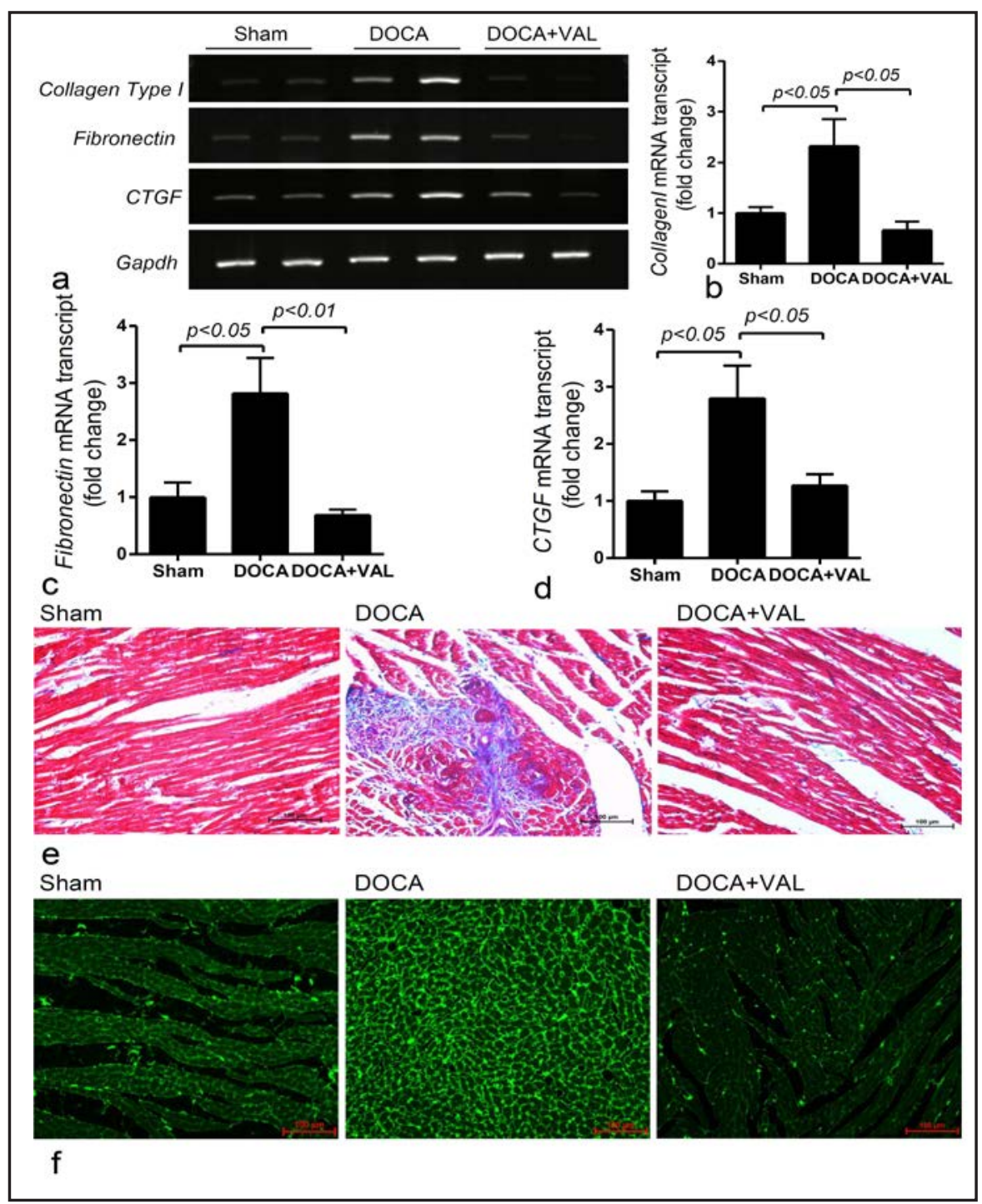

ventricular hypertrophy [13]. To identify whether DOCA-salt hypertensive rats showed fibrotic characteristics, we examined the expression of collagen type I in the left ventricle. DOCA-salt hypertensive rat hearts exhibited an increase in collagen expression that was blocked by treatment with valproate (Fig. 5a and 5b). In addition to collagen, fibronectin is another marker of fibrosis, and the level of fibronectin mRNA was also decreased by valproate treatment (Fig. 5c). Connective tissue growth factor (CTGF) acts as a novel fibrotic mediator [14]. We found that CTGF mRNA levels were increased in DOCA-salt hypertensive heart. These increases were significantly inhibited by treatment with valproate (Fig. $5 \mathrm{~d}$ ). We also performed Masson's trichrome staining to identify interstitial fibrosis in the hearts of DOCA-salt rats. As shown in Fig. 5e, the interstitial fibrosis in DOCA-salt rats at 4 weeks was increased compared with that in sham rats. However, fibrosis was largely reduced in valproate-treated DOCA-salt rats at 4 weeks. We further evaluated the expression of type I collagen by immunohistochemistry after DOCA-salt treatment with or without valproate. The expression of collagen was significantly increased in the myocardial interstitium from DOCA-salt rats compared with sham controls, and this increase was attenuated by valproate (Fig. 5f). 


\section{Kidney Blood Pressure Research}

Kidney Blood Press Res 2013;37:229-239

\begin{tabular}{l|l}
\hline DOI: 10.1159/000350148 & (c) 2013 S. Karger AG, Basel
\end{tabular}

Published online: July 8, 2013

www.karger.com/kbr

Fibrosis

\section{Discussion}

In the present study, we demonstrated that sodium valproate, an HDAC inhibitor, reversed cardiac hypertrophy and fibrosis in DOCA-salt hypertensive rats. Previously, we reported that various HDAC inhibitors including sodium valproate, trichostatin A, SK-7041, and apicidin blocked cardiac hypertrophy in animals models of right ventricular hypertrophy or left ventricular hypertrophy $[2,7,15]$. HDAC is known to have two opposite functions in the heart to inhibit or induce pathological cardiac hypertrophy. Class I HDACs have been shown to induce pathological hypertrophy, whereas class II HDACs block cardiac hypertrophy. It remains unknown which HDAC isoforms act on pathological states in response to a variety of stresses. Some research shows that HDACs respond differently in response to distinct stresses. For example, the catalytic activity of HDAC4 and HDAC6 is induced in spontaneous hypertensive rats and DOCA-salt hypertensive rats, respectively [5, 16]. Another study showed that transient activation of HDAC2 precedes the phenotype of hypertrophic growth in a pressure overload animal model [4].

Cardiac hypertrophy is accompanied by increased heart mass and reactivation of the fetal gene program, such as the ANF and $\beta$-MHC genes. In this study, we also observed increased transcript levels of hypertrophic markers, including $N p p a, N p p b$, and $\beta-M H C$, in the heart of DOCA-salt hypertensive rats. Cardiac transcription factors play a key role in cardiogenesis and are associated with cardiac hypertrophy. GATA4/6, myocyte enhancer factor 2 (MEF2), and homeobox transcription factor Nkx2-5 are known to function in the development of cardiac hypertrophy. We examined whether cardiac-specific transcription factors respond to hypertension stress induced by DOCA-salt. We found that GATA6 expression was increased in DOCA-salt hypertensive rats, whereas the expression of GATA4, MEF2C, and SRF was unchanged.

HDAC is well known to act as an epigenetic gene regulator on histone or nonhistone proteins and may be a target for the treatment of various diseases, such as cancer, kidney disease, stroke, cardiac hypertrophy, and heart failure $[17,18]$. Thus, HDAC inhibitors have emerged as a potential therapeutic target. In this study, we showed an inhibitory effect of valproate on cardiac hypertrophy induced by DOCA-salt hypertension.

In our previous study, treatment with HDAC inhibitors in a cardiac hypertrophy model did not result in changes in HDAC expression. In the present study, class I HDACs including HDAC1, HDAC2, HDAC3, and HDAC8 were not changed in response to chronic hypertension. Class IIa HDACs also showed a similarly unchanged pattern. But, HDAC6, a class IIb HDAC, was induced in the heart of DOCA-salt hypertensive rats. This result is consistent with a recent report by the McKinsey group [5].

Even though the change in HDAC expression was not shown in hypertrophied hearts from DOCA-salt hypertensive rats, activation of HDAC enzyme may be induced in response to chronic hypertension. On the basis of our previous results, we investigated the activity of each class I HDAC. Surprisingly, we found that HDAC2 did not play a key role in the hypertrophied heart of hypertension. By contrast, other class I HDACs such as HDAC8 showed significantly increased HDAC enzyme activity in DOCA-salt hypertension. In addition, the enhanced HDAC8 enzyme activity was inhibited by treatment with valproate. Because of the report of enhanced HDAC6 enzyme activity in chronic hypertension by McKinsey et al, we assessed HDAC6 enzyme activity in our experimental condition. We found that HDAC6 enzyme activity was significantly induced in DOCA-salt hypertension. The mechanism of HDAC8 activation in response to chronic hypertension remains unknown. In this study, we suggest that HDAC isoforms may act differently in response to various stresses. 


\section{Kidney \\ Blood Pressure Research}

Kidney Blood Press Res 2013;37:229-239

DOI: 10.1159/000350148

Published onlıne: July 8, 2013

(C) 2013 S. Karger AG, Basel

www.karger.com/kbr

We recently demonstrated that cardiac fibrosis is inhibited by HDAC inhibitors such as TSA, sodium valproate, and SK-7041 in the hearts of rats in a hypertrophied model with aortic banding or pulmonary artery banding $[2,7]$. In this study, we found that fibrosis was prevented by sodium valproate in the heart of DOCA-salt hypertensive rats via the downregulation of expression of collagen type I, CTGF, and fibronectin. Accumulation of fibrosis is a key characteristic of hypertrophied hearts in hypertensive heart diseases [13]. Consistent with our result, Iyer et al reported that SAHA, an HDAC inhibitor, inhibited interstitial collagen deposition [19].

\section{Conclusion}

We have shown that sodium valproate can regulate the cardiac hypertrophy induced by chronic hypertension via the regulation of HDAC enzyme activity, such as that of HDAC6 and HDAC8. In addition, the HDAC inhibitor used in this study may attenuate cardiac fibrosis by inhibiting fibrosis-associated gene expression. Administration of an HDAC inhibitor may therefore be a potential therapeutic agent for the treatment of pathological cardiac diseases.

\section{Conflicts of interest}

None.

\section{Acknowledgements}

This work was supported by a grant of the Korea Healthcare technology R\&D Project, Ministry for Health, Welfare \& Family Affairs, Republic of Korea (A100317).

\section{References}

1 Gregoretti IV, Lee YM, Goodson HV: Molecular evolution of the histone deacetylase family: functional implications of phylogenetic analysis. J Mol Biol 2004;338:17-31.

-2 Kee HJ, Sohn IS, Nam KI, Park JE, Qian YR, Yin Z, Ahn Y, Jeong MH, Bang YJ, Kim N, Kim JK, Kim KK, Epstein JA, Kook H: Inhibition of histone deacetylation blocks cardiac hypertrophy induced by angiotensin II infusion and aortic banding. Circulation 2006;113:51-59.

-3 Kee HJ, Kwon JS, Shin S, Ahn Y, Jeong MH, Kook H: Trichostatin A prevents neointimal hyperplasia via activation of Kruppel like factor 4. Vascul Pharmacol 2011;55:127-134.

-4 Kee HJ, Eom GH, Joung H, Shin S, Kim JR, Cho YK, Choe N, Sim BW, Jo D, Jeong MH, Kim KK, Seo JS, Kook H: Activation of histone deacetylase 2 by inducible heat shock protein 70 in cardiac hypertrophy. Circ Res 2008;103:1259-1269.

-5 Lemon DD, Horn TR, Cavasin MA, Jeong MY, Haubold KW, Long CS, Irwin DC, McCune SA, Chung E, Leinwand LA, McKinsey TA: Cardiac HDAC6 catalytic activity is induced in response to chronic hypertension. J Mol Cell Cardiol 2011;51:41-50.

-6 Lorell BH, Carabello BA: Left ventricular hypertrophy: pathogenesis, detection, and prognosis. Circulation 2000;102:470-479.

7 Cho YK, Eom GH, Kee HJ, Kim HS, Choi WY, Nam KI, Ma JS, Kook H: Sodium valproate, a histone deacetylase inhibitor, but not captopril, prevents right ventricular hypertrophy in rats. Circ J 2010;74:760-770.

8 Kee HJ, Kook H: Kruppel-like factor 4 mediates histone deacetylase inhibitor-induced prevention of cardiac hypertrophy. J Mol Cell Cardiol 2009;47:770-780. 


\section{Kidney \\ Blood Pressure Research}

Kidney Blood Press Res 2013;37:229-239

\section{DOI: 10.1159/000350148}

Published online: July 8, 2013

(C) 2013 S. Karger AG, Basel

www.karger.com/kbr

Kee/Bae/Park/Lee/Suh/Kim/Jeong: Valproic Acid Inhibits Cardiac Hypertrophy and Fibrosis

-9 Bae EH, Kim IJ, Ma SK, Kim SW: Rosiglitazone prevents the progression of renal injury in DOCA-salt hypertensive rats. Hypertens Res 2010;33:255-262.

10 Kee HJ, Kim JR, Nam KI, Park HY, Shin S, Kim JC, Shimono Y, Takahashi M, Jeong MH, Kim N, Kim KK, Kook $\mathrm{H}$ : Enhancer of polycomb1, a novel homeodomain only protein-binding partner, induces skeletal muscle differentiation. J Biol Chem 2007;282:7700-7709.

11 Bae EH, Kim IJ, Park JW, Ma SK, Lee JU, Kim SW: Renoprotective effect of rosuvastatin in DOCA-salt hypertensive rats. Nephrol Dial Transplant 2010;25:1051-1059.

$\checkmark 12$ Akazawa H, Komuro I: Roles of cardiac transcription factors in cardiac hypertrophy. Circ Res 2003;92:1079-1088.

13 Diez J: Mechanisms of cardiac fibrosis in hypertension. J Clin Hypertens (Greenwich) 2007; 9:546-550.

-14 Koitabashi N, Arai M, Niwano K, Watanabe A, Endoh M, Suguta M, Yokoyama T, Tada H, Toyama T, Adachi H, Naito S, Oshima S, Nishida T, Kubota S, Takigawa M, Kurabayashi M: Plasma connective tissue growth factor is a novel potential biomarker of cardiac dysfunction in patients with chronic heart failure. Eur J Heart Fail 2008;10:373-379.

15 Eom GH, Cho YK, Ko JH, Shin S, Choe N, Kim Y, Joung H, Kim HS, Nam KI, Kee HJ, Kook H: Casein kinase2alpha1 induces hypertrophic response by phosphorylation of histone deacetylase 2 S394 and its activation in the heart. Circulation 2011;123:2392-2403.

16 Usui T, Okada M, Mizuno W, Oda M, Ide N, Morita T, Hara Y, Yamawaki H: HDAC4 mediates development of hypertension via vascular inflammation in spontaneous hypertensive rats. Am J Physiol Heart Circ Physiol 2012;302:H1894-1904.

17 Lv L, Tang YP, Han X, Wang X, Dong Q: Therapeutic application of histone deacetylase inhibitors for stroke. Cent Nerv Syst Agents Med Chem 2011;11:138-149.

18 McKinsey TA: Targeting inflammation in heart failure with histone deacetylase inhibitors. Mol Med 2011;17:434-441.

19 Iyer A, Fenning A, Lim J, Le GT, Reid RC, Halili MA, Fairlie DP, Brown L: Antifibrotic activity of an inhibitor of histone deacetylases in DOCA-salt hypertensive rats. Br J Pharmacol 2010;159:1408-1417. 